\title{
An inverse seesaw model with $U(1)_{R}$ gauge symmetry
}

\author{
Takaaki Nomura, ${ }^{1}$ Hiroshi Okada, ${ }^{2}$ \\ ${ }^{1}$ School of Physics, KIAS, Seoul 02455, Republic of Korea \\ ${ }^{2}$ Asia Pacific Center for Theoretical Physics, Pohang, Geyoengbuk 790-784, Republic of Korea
}

\begin{abstract}
We propose a natural realization of the inverse seesaw model with right-handed and flavor dependent $U(1)$ gauge symmetries, in which we formulate the neutrino mass matrix to reproduce current neutrino oscillation data in a general way. Also we study a possibility to provide predictions to the neutrino sector by imposing an additional flavor dependent $U(1)_{L_{\mu}-L_{\tau}}$ gauge symmetry that also satisfies the gauge anomaly cancellation conditions associated with $U(1)_{R}$. Then we analyze collider physics on an extra gauge boson and show a possibility of detection.
\end{abstract}

\section{DOI: 10.31526/LHEP.2.2018.01}

$U(1)_{B-L}$ [1] and $U(1)_{R}$ [2, 3, 4, 5, 6] gauge symmetries require three families of neutral right-handed (or left-handed) fermions in order to cancel the gauge anomalies. These two symmetries have the feature that they are very similar to each other in the Yukawa sector. In fact, once a lepton Yukawa model is constructed in a gauge symmetry, the other symmetry can also reproduce the same one. And these symmetries are known as a natural extension of the standard model (SM) to realize various seesaw mechanisms such as canonical seesaw model [7, 8, 9, 10], inverse seesaw model [11, 12], linear seesaw model [12, 13, 14], etc.

On the other hand, the nature of these two gauge sectors are so different, and one might be able to test the differences via current or future experiments so as to make use of the polarized electron/positron beam at e.g., ILC [15]. Indeed, $U(1)_{B-L}$ is chirality-universal in the kinetic terms, while $U(1)_{R}$ has righthanded chirality only. In this sense, it would be worthwhile for us to construct models with gauged $U(1)_{B-L}$ and/or $U(1)_{R}$ symmetry as many as possible, so that we can distinguish these two extra symmetries in various phenomenological points of view.

In this paper, we construct an inverse seesaw model with $U(1)_{R}$ symmetry, in which we formulate the neutrino mass matrix to reproduce current neutrino oscillation data [16] in a general way. Inverse seesaw requires a left-handed neutral fermions $N_{L}$ in addition to the right-handed ones $N_{R}$, and provides us more complicated neutrino mass matrix. Therefore, each of mass hierarchies are softer than the other models such as canonical seesaw and it could provide abundant phenomenologies such as unitarity constraints. Note that we expect $N_{L}$ has nonzero $U(1)_{B-L}$ charge because it is a kind of partner of $N_{R}$. In that case, however, $U(1)_{B-L}$ can not be gauged since anomaly cancellation condition can not be satisfied. Therefore,introducing left-handed singlet fermion is more natural in gauged $U(1)_{R}$ symmetry case compared with gauged $U(1)_{B-L}$ symmetry case since the left-handed singlet fermion cannot have lepton number in the latter case. Also, we study a possibility to provide predictions to the neutrino sector by imposing an additional flavor dependent $U(1)_{L_{\mu}-L_{\tau}}$ gauge symmetry that also satisfies the gauge anomaly cancellations among $U(1)_{R} \cdot{ }^{1}$ Then we analyze collider physics on an extra gauge boson and show a possibility of detection.

${ }^{1} U(1)_{B-L} \times U(1)_{L_{\mu}-L_{\tau}}$ can also be anomaly free. See ref. [17.

\begin{tabular}{|c||c|c|c|c|c|c|c||c|c|}
\hline & $Q_{L}^{a}$ & $u_{R}^{a}$ & $d_{R}^{a}$ & $L_{L}^{a}$ & $e_{R}^{a}$ & $N_{R}^{a}$ & $S_{L}^{a}$ & $H$ & $\varphi_{1(2)}$ \\
\hline$S U(3)_{C}$ & $\mathbf{3}$ & $\mathbf{3}$ & $\mathbf{3}$ & $\mathbf{1}$ & $\mathbf{1}$ & $\mathbf{1}$ & $\mathbf{1}$ & $\mathbf{1}$ & $\mathbf{1}$ \\
\hline$S U(2)_{L}$ & $\mathbf{2}$ & $\mathbf{1}$ & $\mathbf{1}$ & $\mathbf{2}$ & $\mathbf{1}$ & $\mathbf{1}$ & $\mathbf{1}$ & $\mathbf{2}$ & $\mathbf{1}$ \\
\hline$U(1)_{Y}$ & $\frac{1}{6}$ & $\frac{2}{3}$ & $-\frac{1}{3}$ & $-\frac{1}{2}$ & -1 & 0 & 0 & $\frac{1}{2}$ & 0 \\
\hline$U(1)_{R}$ & 0 & 1 & -1 & 0 & -1 & 1 & 0 & 1 & $1(0)$ \\
\hline
\end{tabular}

TABLE 1: Charge assignments of the fields under $S U(3)_{C} \times$ $S U(2)_{L} \times U(1)_{Y} \times U(1)_{R}$, where the upper index $a$ is the number of family that runs over 1-3. Singlet scalar $\varphi_{2}$ is required when we add $U(1)_{L_{\mu}-L_{\tau}}$ gauge symmetry.

This letter is organized as follows: We review our model and formulate the lepton sector. Then we discuss phenomenologies of neutrinos and an extra neutral gauge boson at colliders. Finally we devote the summary of our results and the conclusion.

At first, we add three families of right(left)-handed fermions $N_{R}\left(S_{L}\right)$ with $1(0)$ charge under the $U(1)_{R}$ gauge symmetry, and an isospin singlet boson $\varphi_{1}$ with 1 charge under the same symmetry. Here we denote each of vacuum expectation value to be $\langle H\rangle \equiv v_{H} / \sqrt{2}$, and $\left\langle\varphi_{1}\right\rangle \equiv v_{\varphi_{1}} / \sqrt{2}$. Furthermore, the SM Higgs boson $H$ also has 1 charge to induce the masses of SM fermions from the Yukawa Lagrangian after the spontaneously symmetry breaking. ${ }^{2}$ All the field contents and their assignments are summarized in Table 1 The relevant Yukawa Lagrangian under these symmetries is given by

$$
\begin{aligned}
-\mathcal{L}_{\ell}= & y_{\ell a a} \bar{L}_{L}^{a} H e_{R}^{a}+y_{D_{a b}} \bar{L}_{L}^{a} \tilde{H} N_{R}^{b}+y_{S N_{a a}} \bar{S}_{L}^{a} N_{R}^{a} \varphi_{1}^{*} \\
& +\mu_{a b} \bar{S}_{L}^{a}\left(S_{L}^{c}\right)^{b}+\text { h.c., }
\end{aligned}
$$

where $\tilde{H} \equiv i \sigma_{2} H$, and upper indices $(a, b)=1-3$ are the number of families, and $y_{\ell}$ and $y_{S N}$ can be diagonal matrix without loss of generality due to the redefinitions of the fermions. Each of the mass matrix is defined by $m_{\ell}=y_{\ell} v / \sqrt{2}, m_{D}=y_{D} v_{H} / \sqrt{2}$, and $M_{S N}=y_{S N} v_{\varphi_{1}} / \sqrt{2}$.

After the spontaneously symmetry breaking, neutral fermion mass matrix with $9 \times 9$ is given by

$$
M_{N}=\left[\begin{array}{ccc}
0 & m_{D} & 0 \\
m_{D}^{T} & 0 & M_{S N}^{T} \\
0 & M_{S N} & \mu
\end{array}\right] .
$$

\footnotetext{
${ }^{2}$ Due to the feature of nonzero charges of $H$, lower bound on the breaking scale of $U(1)_{R}$ is determined via the precision test of $Z$ boson mass; $\Lambda \gtrsim \mathcal{O}(10)$ $\mathrm{TeV}$ 5.

${ }^{3}$ Since the quark sector is exactly same as the one of SM, we neglect it hereafter.
} 
Then the active neutrino mass matrix can approximately be found as

$$
m_{v} \approx m_{D} M_{S N}^{-1} \mu\left(M_{S N}^{T}\right)^{-1} m_{D}^{T}
$$

where $\mu \ll m_{D} \lesssim M_{S N}$ is expected ${ }^{4}$ The neutrino mass matrix is diagonalized by unitary matrix $U_{M N S} ; D_{v}=U_{M N S}^{T} m_{v} U_{M N S}$, where $D_{v} \equiv \operatorname{diag}\left(m_{1}, m_{2}, m_{3}\right)$. One of the elegant ways to reproduce the current neutrino oscillation data [16] is to apply the Casas-Ibarra parametrization [18] without loss of generality, and find the following relation

$$
m_{D}=U_{M N S}^{*} \sqrt{D_{\nu}} O_{\text {mix }} \sqrt{I_{N}}\left(L_{N}^{T}\right)^{-1} .
$$

Here $O_{\text {mix }}$ is an arbitrary 3 by 3 orthogonal matrix with complex values, $I_{N}$ is a diagonal matrix, and $L_{N}$ is a lower unit triangular [30], which can uniquely be decomposed to be $M_{S N}^{-1} \mu\left(M_{S N}^{T}\right)^{-1}=L_{N}^{T} I_{N} L_{N}$, since it is symmetric matrix. Note here that all the components of $m_{D}$ should not exceed $246 \mathrm{GeV}$, once perturbative limit of $y_{D}$ is taken to be 1 .

Now we introduce local $U(1)_{L_{\mu}-L_{\tau}}$ symmetry to restrict neutrino mass structure in inverse seesaw scenario [20, 19] where we add SM singlet scalar $\varphi_{2}$ with $L_{\mu}-L_{\tau}$ charge 1 to break the symmetry spontaneously. Then Yukawa interactions and Majorana masses are constrained, and we have new Yukawa interactions;

$$
-\mathcal{L}_{\text {new }}=y_{i j} \varphi_{2} \bar{S}_{L}^{i}\left(S_{L}^{c}\right)^{j}+\text { h.c. }
$$

where index $i(j)$ is determined to satisfy gauge invariance. Thus once we impose $U(1)_{L_{\mu}-L_{\tau}}$ gauge symmetry as shown in table $22^{5}$ the mass matrices $m_{D}, M_{S N}, \mu$ are specified to be

$$
\begin{aligned}
M_{S N} & =\left[\begin{array}{ccc}
m_{S N_{1}} & 0 & 0 \\
0 & m_{S N_{2}} & 0 \\
0 & 0 & m_{S N_{3}}
\end{array}\right], \\
m_{D} & =\left[\begin{array}{ccc}
m_{d_{1}} & 0 & 0 \\
0 & m_{d_{2}} & 0 \\
0 & 0 & m_{d_{3}}
\end{array}\right], \quad \mu=\left[\begin{array}{ccc}
\mu_{1} & \mu_{2} & \mu_{3} \\
\mu_{2} & 0 & \mu_{4} \\
\mu_{3} & \mu_{4} & 0
\end{array}\right],
\end{aligned}
$$

where $\mu_{2,3}$ is induced only after the $U(1)_{L_{\mu}-L_{\tau}}$ spontaneously symmetry breaking. Therefore, the neutrino mass matrix directly reflects the form of $\mu$ as

$$
m_{v}=\left[\begin{array}{ccc}
\mu_{1} \frac{m_{d_{1}}^{2}}{m_{S N_{1}}^{2}} & \mu_{2} \frac{m_{d_{1}} m_{d_{2}}}{m_{S N_{1}} m_{S N_{2}}} & \mu_{3} \frac{m_{d_{1}} m_{d_{3}}}{m_{S N_{1}} m_{S N_{3}}} \\
\mu_{2} \frac{m_{d_{1}} m_{d_{2}}}{m_{S N_{1}} m_{S N_{2}}} & 0 & \mu_{4} \frac{m_{d_{2}} m_{d_{3}}}{m_{S N_{2}} m_{S N_{3}}} \\
\mu_{3} \frac{m_{d_{1}} m_{d_{3}}}{m_{S N_{1}} m_{S N_{3}}} & \mu_{4} \frac{m_{d_{2}} m_{d_{3}}}{m_{S N_{2}} m_{S N_{3}}} & 0
\end{array}\right] .
$$

Thus we can predict inverted neutrino ordering and specific value of Dirac phase by analyzing the two-zero texture [21, 19]. Here the number of parameters in the neutrino mass matrix is nine real parameters (that are equivalent of four complexes and one real). Then one more phase is there in addition to the Dirac phase and two Majorana phases. Note here that this twozero texture originates from $\mu$ in the inverse seesaw model that cannot be reproduced by a canonical seesaw model.

\footnotetext{
${ }^{4}$ These hierarchies could be explained by several mechanisms such as radiative models [31 32,33 and effective models with higher order terms 34 .

${ }^{5}$ Before the discussion of neutrino sector, we have to check the gauge anomalies. The non-trivial gauge anomalies are $\left[U(1)_{R}\right]^{2} U(1)_{L_{\mu}-L_{\tau}}$ and $U(1)_{R}\left[U(1)_{L_{\mu}-L_{\tau}}\right]^{2}$, and one straightforwardly confirms that there are no anomalies in our field assignments.
}

\begin{tabular}{|c|c|c|}
\hline \hline & $L_{L^{\prime}}^{2}, N_{R^{\prime}}^{2}, S_{L^{\prime}}^{2}, e_{R^{\prime}}^{2}, \varphi_{2}$ & $L_{L^{\prime}}^{3} N_{R^{\prime}}^{3}, S_{L}^{3}, e_{R}^{3}$ \\
\hline \hline$U(1)_{L_{\mu}-L_{\tau}}$ & 1 & -1 \\
\hline
\end{tabular}

TABLE 2: Charge assignments of the our fields under $U(1)_{L_{\mu}-L_{\tau}}$, where the upper index $a$ is the number of family that runs over $1-3$. The other fields do not have $L_{\mu}-L_{\tau}$ charge.

Let us briefly discuss non-unitarity matrix $U_{M N S}^{\prime}$. This is typically parametrized by the form

$$
U_{M N S}^{\prime} \equiv\left(1-\frac{1}{2} F F^{+}\right) U_{M N S}
$$

where $F \equiv\left(m_{S N}^{T}\right)^{-1} m_{D}$ is a hermitian matrix, and $U_{M N S}^{\prime}$ represents the deviation from the unitarity. The global constraints are found via several experimental results such as the SM $W$ boson mass $M_{W}$, the effective Weinberg angle $\theta_{W}$, several ratios of $Z$ boson fermionic decays, invisible decay of $Z$, electroweak universality, measured Cabbibo-Kobayashi-Maskawa, and lepton flavor violations [35]. The result is then given by [36]

$$
\left|F F^{\dagger}\right| \leq\left[\begin{array}{lll}
2.5 \times 10^{-3} & 2.4 \times 10^{-5} & 2.7 \times 10^{-3} \\
2.4 \times 10^{-5} & 4.0 \times 10^{-4} & 1.2 \times 10^{-3} \\
2.7 \times 10^{-3} & 1.2 \times 10^{-3} & 5.6 \times 10^{-3}
\end{array}\right]
$$

Once we conservatively take $F \approx 10^{-5}$, we find $\mu \approx 1-10 \mathrm{GeV}$ to satisfy the typical neutrino mass scale, which can be easy to realize.

Here we discuss collider physics of our model, in particular we focus on $Z_{R}^{\prime}$ boson from $U(1)_{R}$ which obtains its mass via the vacuum expectation value of $\varphi_{2}$. The gauge interaction associated with $Z_{R}^{\prime}$ is given by

$$
\mathcal{L} \supset g_{R}\left(\bar{u}_{R} \gamma_{\mu} u_{R}-\bar{d}_{R} \gamma_{\mu} d_{R}-\bar{\ell}_{R} \gamma_{\mu} \ell_{R}+\bar{N}_{R} \gamma_{\mu} N_{R}\right) Z_{R}^{\prime \mu}
$$

where $g_{R}$ is gauge coupling constant for $U(1)_{R}$, and flavor index is omitted.

$Z_{R}^{\prime}$ physics at the LHC: In our model $Z_{R}^{\prime}$ can be produced via $q \bar{q} \rightarrow Z_{R}^{\prime}$ process and it will decay into SM fermions and $N_{R}$ if kinematically allowed. Then stringent constraint is given by di-lepton resonance search at the LHC. We estimate the cross section with CalcHEP [23] by the use of the CTEQ6 parton distribution functions (PDFs) [24] and implementing relevant interactions. In addition, we find branching ratio (BR) for the decay mode $Z_{R}^{\prime} \rightarrow e^{+} e^{-} / \mu^{+} \mu^{-}$is $\sim 4.8 \%$ for both electron and muon when we assume $\bar{N}_{R} N_{R}$ mode is not kinematically allowed; even if we include $\bar{N}_{R} N_{R}$ mode the BR does not change much as $B R\left(Z_{R}^{\prime} \rightarrow e^{+} e^{-} / \mu^{+} \mu^{-}\right) \gtrsim 4.2 \%$. In Fig. 1. we show $\sigma\left(p p \rightarrow Z_{R}^{\prime}\right) B R\left(Z_{R}^{\prime} \rightarrow \ell^{+} \ell^{-}\right)$as a function of $m_{Z_{R}^{\prime}}$ for several values of $g_{R}$ where the $B R$ is sum of electron and muon mode and the red curve indicates the LHC limit obtained from ref. [25]. We find that $Z_{R}^{\prime}$ mass should be heavier than $\sim 3.8 \mathrm{TeV}$ for $g_{R}=0.1$ where corresponding production cross section is $\sigma\left(p p \rightarrow Z_{R}^{\prime}\right) \lesssim 1 \mathrm{fb}$.

Now we discuss the production of heavy neutrino $v_{H}$ at the LHC via $Z_{R}^{\prime}$ boson. If masses of $v_{H_{i}}$ are sufficiently lighter than $m_{Z_{R}^{\prime}} / 2, B R\left(Z_{R}^{\prime} \rightarrow v_{H} v_{H}\right)$ is around $4 \%$ for each mass eigenstate. Then $v_{H}$ decays such that $v_{H} \rightarrow W^{ \pm} \ell^{\mp}$ and $v_{H} \rightarrow Z v_{L}$ via mixing in neutrino sector. As we have discussed above, $Z_{R}^{\prime}$ production cross section is less than $\sim 1 \mathrm{fb}$ for $m_{Z_{R}^{\prime}}$ being several 


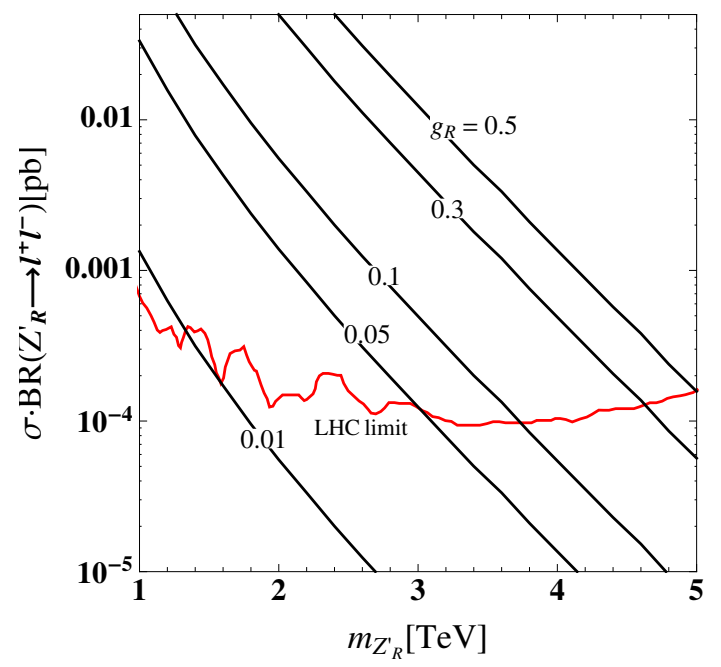

FIGURE 1: The product of $Z_{R}^{\prime}$ production cross section and $B R\left(Z_{R}^{\prime} \rightarrow \ell^{+} \ell^{-}\right)$where region above red curve is excluded by the latest data [25].

\begin{tabular}{|c||c|c|c|}
\hline \hline Mode & $\ell^{-} \ell^{+}$ & $q \bar{q}$ & $\nu_{H} \bar{v}_{H}$ \\
\hline \hline$B R$ & 0.042 & 0.13 & 0.042 \\
\hline
\end{tabular}

TABLE 3: The BRs for $Z_{R}^{\prime}$ decay under an approximation assuming $m_{Z_{R}^{\prime}}^{2}>>m_{f}^{2}$ where $m_{f}$ is mass of final state fermion.

$\mathrm{TeV}$ scale, and $v_{H}$ production cross section will be $\sigma \cdot B R \lesssim 0.04$ $\mathrm{fb}$ for each mass eigenstate. Thus large integrated luminosity is required to obtain sufficient number of events to analyze the signal. It is also important to confirm the ratio of the $B R$ of each decay mode of $Z_{R}^{\prime}$ to distinguish it from other $Z^{\prime}$ boson like that from $U(1)_{B-L}$ gauge symmetry where the approximated $B R$ s are given in Table. 3

Here we also comment on $Z_{\mu-\tau}^{\prime}$ boson from $U(1)_{L_{\mu}-L_{\tau}}$ gauge symmetry. It is difficult to detect $Z_{\mu-\tau}^{\prime}$ when we consider it to be light as $\mathcal{O}(10)-\mathcal{O}(100) \mathrm{MeV}$ so that muon $g-2$ can be explained [22]. The $Z_{\mu-\tau}^{\prime}$ interaction induces flavor violating decay of heavy neutrino such as $v_{H_{i}} \rightarrow Z_{\mu-\tau}^{\prime} \nu_{H_{j}}$ where $m_{v_{H_{i}}}>m_{v_{H_{j}}}$. Thus phenomenology of heavy neutrino at the LHC would be affected by the gauge boson. However, detailed analysis is beyond the scope of this paper.

$Z_{R}^{\prime}$ physics at lepton collider: Although it would be difficult to produce $Z_{R}^{\prime}$ directly at lepton colliders we can explore the effective interaction induced from $Z_{R}^{\prime}$ exchange;

$$
\mathcal{L}_{e f f}=\frac{1}{1+\delta_{e \ell}} \frac{g_{R}^{2}}{m_{Z_{R}^{\prime}}^{2}}\left(\bar{e} \gamma^{\mu} P_{R} e\right)\left(\bar{f} \gamma_{\mu} P_{R} f\right),
$$

where $f$ indicates all the fermions in the model, and only the right-handed chirality appears due to the nature of $U(1)_{R}$ symmetry. For example, the analysis of data by LEP experiment in ref. [26] provides the constraint $\frac{m_{Z_{R}^{\prime}}}{g_{R}} \gtrsim 3.7 \mathrm{TeV}$.

Furthermore, chirality structure of the effective interaction could be tested by measuring the process $e^{+} e^{-} \rightarrow f \bar{f}$ at the International Linear Collider (ILC) using polarized initial state.
The partially-polarized differential cross section can be defined as [27]

$$
\frac{d \sigma\left(P_{e^{-}}, P_{e^{+}}\right)}{d \cos \theta}=\sum_{\sigma_{e^{-}}, \sigma_{e^{+}}= \pm} \frac{1+\sigma_{e^{-}} P_{e^{-}}}{2} \frac{1+\sigma_{e^{+}} P_{e^{-}}}{2} \frac{d \sigma_{\sigma_{e^{-}} \sigma_{e^{+}}}}{d \cos \theta}
$$

where $P_{e^{-}\left(e^{+}\right)}$is the degree of polarization for the electron(positron) beam and $\sigma_{\sigma_{e}-\sigma_{e}+}$ indicates the cross section when the helicity of initial electron(positron) is $\sigma_{e^{-}}\left(e^{+}\right)$and the helicity of final states is summed up; more detailed form is found in ref [27]. The polarized cross sections $\sigma_{L, R}$ is given by following two cases as realistic values at the ILC [28]:

$$
\frac{d \sigma_{R}}{d \cos \theta}=\frac{d \sigma(0.8,-0.3)}{d \cos \theta}, \quad \frac{d \sigma_{L}}{d \cos \theta}=\frac{d \sigma(-0.8,0.3)}{d \cos \theta} .
$$

Then we apply $\sigma_{R}$ to study the sensitivity to $Z_{R}^{\prime}$ since it is sensitive to right-handed current interactions [5]. To investigate the effect of the new interaction we consider the measurement of a forward-backward asymmetry at the ILC which is given by

$$
\begin{aligned}
& A_{F B}=\frac{N_{F}-N_{B}}{N_{F}+N_{B}}, \\
& N_{F(B)}=\epsilon L \int_{0\left(-c_{\max }\right)}^{c_{\max }(0)} d \cos \theta \frac{d \sigma}{d \cos \theta},
\end{aligned}
$$

where a kinematical cut $c_{\max }=0.5(0.95)$ is chosen to maximize the sensitivity for electron(muon) [29], $L$ is an integrated luminosity and $\epsilon$ is an efficiency depending on the final states which is assumed to be $\epsilon=1$ for electron and muon final states. The sensitivity to $Z_{R}^{\prime}$ contribution is estimated by

$$
\Delta A_{F B}=\left|A_{F B}^{S M+Z_{R}^{\prime}}-A_{F B}^{S M}\right|,
$$

where $A_{F B}^{S M+Z_{R}^{\prime}}$ and $A_{F B}^{S M}$ are forward-backward asymmetry for "SM $+Z_{R}^{\prime \prime}$ " and $\mathrm{SM}$ cases, respectively. We compare $\Delta A_{F B}$ with a statistical error of the asymmetry in only SM case

$$
\delta_{A_{F B}}^{S M}=\sqrt{\frac{1-\left(A_{F B}^{S M}\right)^{2}}{N_{F}^{S M}+N_{B}^{S M}}},
$$

and we focus on muon final state which is the most sensitive one. We find that it is difficult to get $\Delta A_{F B}>\delta_{A_{F B}}^{S M}$ for $\sqrt{s}=$ 250 or $500 \mathrm{GeV}$ in the region which satisfies the LHC constraint even if the integrated luminosity is $\mathcal{O}(10) \mathrm{ab}^{-1}$. On the other hand, for $\sqrt{s}=1 \mathrm{TeV}, \Delta A_{F B} \sim 2 \delta_{A_{F B}}^{S M}$ can be obtained with the integrated luminosity of $5 \mathrm{ab}^{-1}$ with $m_{Z_{R}^{\prime}} / g_{R}=40 \mathrm{TeV}$. Therefore to investigate the chirality structure, we need $\sqrt{s}=1$ $\mathrm{TeV}$ with large integrated luminosity which would be achieved if the ILC is upgraded [15].

We have constructed an inverse seesaw model with $U(1)_{R}$ symmetry, in which we have formulated the neutrino mass matrix to reproduce current neutrino oscillation data in a general way. Also we have found a predictive two-zero neutrino mass matrix, by imposing an additional flavor dependent $U(1)_{L_{\mu}-L_{\tau}}$ gauge symmetry that also satisfies the gauge anomaly cancellations among $U(1)_{R}$. Then we have analyzed collider physics on an extra gauge boson and have shown a possibility of detection. Although, the result of collider physics is almost the same as the one of our canonical seesaw model [5]. the neutrino predictions originate from the inverse seesaw model that could be difficult to reproduce any canonical seesaw models. 


\section{ACKNOWLEDGMENTS}

H. O. is sincerely grateful for KIAS and all the members.

\section{References}

[1] R. N. Mohapatra and R. E. Marshak, Phys. Rev. Lett. 44, 1316 (1980) Erratum: [Phys. Rev. Lett. 44, 1643 (1980)].

[2] S. Jung, H. Murayama, A. Pierce and J. D. Wells, Phys. Rev. D 81, 015004 (2010) [arXiv:0907.4112 [hep-ph]].

[3] P. Ko, Y. Omura and C. Yu, JHEP 1401, 016 (2014) [arXiv:1309.7156 [hep-ph]].

[4] T. Nomura and H. Okada, Phys. Lett. B 761, 190 (2016) [arXiv:1606.09055 [hep-ph]].

[5] T. Nomura and H. Okada, Phys. Rev. D 97, no. 1, 015015 (2018) [arXiv:1707.00929 [hep-ph]].

[6] W. Chao, Eur. Phys. J. C 78, no. 2, 103 (2018) [arXiv:1707.07858 [hep-ph]].

[7] P. Minkowski, Phys. Lett. B 67, 421 (1977);

[8] T. Yanagida, in Proceedings of the Workshop on the Unified Theory and the Baryon Number in the Universe (O. Sawada and A. Sugamoto, eds.), KEK, Tsukuba, Japan, 1979, p. 95;

[9] M. Gell-Mann, P. Ramond, and R. Slansky, Supergravity (P. van Nieuwenhuizen et al. eds.), North Holland, Amsterdam, 1979, p. 315; S. L. Glashow, The future of elementary particle physics, in Proceedings of the 1979 Cargèse Summer Institute on Quarks and Leptons (M. Levy et al. eds.), Plenum Press, New York, 1980, p. 687;

[10] R. N. Mohapatra and G. Senjanovic, Phys. Rev. Lett. 44, 912 (1980).

[11] R. N. Mohapatra and J. W. F. Valle, Phys. Rev. D 34, 1642 (1986).

[12] D. Wyler and L. Wolfenstein, Nucl. Phys. B 218, 205 (1983).

[13] E. K. Akhmedov, M. Lindner, E. Schnapka and J. W. F. Valle, Phys. Lett. B 368, 270 (1996) [hepph/9507275].

[14] E. K. Akhmedov, M. Lindner, E. Schnapka and J. W. F. Valle, Phys. Rev. D 53, 2752 (1996) [hepph/9509255].

[15] T. Barklow, J. Brau, K. Fujii, J. Gao, J. List, N. Walker and K. Yokoya, arXiv:1506.07830 [hep-ex].

[16] C. Patrignani et al. [Particle Data Group], Chin. Phys. C 40, no. 10, 100001 (2016).

[17] T. Araki, J. Heeck and J. Kubo, JHEP 1207, 083 (2012) [arXiv:1203.4951 [hep-ph]].

[18] J. A. Casas and A. Ibarra, Nucl. Phys. B 618, 171 (2001) [hep-ph/0103065].

[19] A. Dev, arXiv:1710.02878 [hep-ph].

[20] A. Biswas, S. Choubey and S. Khan, arXiv:1805.00568 [hep-ph].

[21] H. Fritzsch, Z. z. Xing and S. Zhou, JHEP 1109, 083 (2011) [arXiv:1108.4534 [hep-ph]].

[22] S. N. Gninenko and N. V. Krasnikov, Phys. Lett. B 513, 119 (2001) [hep-ph/0102222].

[23] A. Belyaev, N. D. Christensen and A. Pukhov, Comput. Phys. Commun. 184, 1729 (2013) [arXiv:1207.6082 [hepph]].

[24] P. M. Nadolsky, H. L. Lai, Q. H. Cao, J. Huston, J. Pumplin, D. Stump, W. K. Tung and C.-P. Yuan, Phys. Rev. D 78, 013004 (2008) [arXiv:0802.0007 [hep-ph]].

[25] M. Aaboud et al. [ATLAS Collaboration], JHEP 1710, 182 (2017) [arXiv:1707.02424 [hep-ex]].
[26] S. Schael et al. [ALEPH and DELPHI and L3 and OPAL and LEP Electroweak Collaborations], Phys. Rept. 532, 119 (2013) [arXiv:1302.3415 [hep-ex]].

[27] T. Nomura, H. Okada and H. Yokoya, Nucl. Phys. B 929, 193 (2018) [arXiv:1702.03396 [hep-ph]].

[28] H. Baer et al., arXiv:1306.6352 [hep-ph].

[29] T. H. Tran, V. Balagura, V. Boudry, J. C. Brient and H. Videau, Eur. Phys. J. C 76, no. 8, 468 (2016) [arXiv:1510.05224 [physics.ins-det]].

[30] S. Baek, H. Okada and Y. Orikasa, arXiv:1703.00685 [hepph].

[31] P. S. B. Dev and A. Pilaftsis, "Minimal Radiative Neutrino Mass Mechanism for Inverse Seesaw Models," Phys. Rev. D 86, 113001 (2012) [arXiv:1209.4051 [hep-ph]].

[32] P. S. Bhupal Dev and A. Pilaftsis, "Light and Superlight Sterile Neutrinos in the Minimal Radiative Inverse Seesaw Model," Phys. Rev. D 87 (2013) no.5, 053007 [arXiv:1212.3808 [hep-ph]].

[33] A. Das, T. Nomura, H. Okada and S. Roy, Phys. Rev. D 96, no. 7, 075001 (2017) [arXiv:1704.02078 [hep-ph]].

[34] H. Okada and T. Toma, Phys. Rev. D 86, 033011 (2012) [arXiv:1207.0864 [hep-ph]].

[35] E. Fernandez-Martinez, J. Hernandez-Garcia and J. LopezPavon, JHEP 1608, 033 (2016) [arXiv:1605.08774 [hep-ph]].

[36] N. R. Agostinho, G. C. Branco, P. M. F. Pereira, M. N. Rebelo and J. I. Silva-Marcos, arXiv:1711.06229 [hep-ph]. 